\title{
Dynamic Coating Capillary Electrophoresis for Separation of Humic Acid Using Mixture Solution of Non-ionic Polymers both as Coating Agent and Separation Medium
}

\author{
Toru TaKaHASHI, ${ }^{* \dagger}$ Jun KaWAna, $* *$ Yuuki TAMURA, $* *$ and Hitoshi HoShINO** \\ *Graduate School of Engineering, University of Fukui, 3-9-1 Bunkyo, Fukui 910-8507, Japan \\ **Graduate School of Environmental Studies, Tohoku University, 20 Aoba, Aramaki, Aoba, \\ Sendai 980-8579, Japan
}

\begin{abstract}
A simple, rapid, and precise dynamic coating capillary electrophoretic separation method for water-soluble humic substances is proposed. An aqueous solution containing hydroxyethyl cellulose (HEC), polyethylene glycol (PEG), and $\mathrm{pH}$ buffer component was employed for both the dynamic coating agent and the separation medium. The procedure for the coating of the capillary inner wall was simply filling the buffered polymer mixture solution into the capillary that had been treated with $1 \mathrm{M}$ aqueous $\mathrm{HCl}$ solution. The solution for the capillary coating was directly used as the electrophoretic buffer solution for CE separation. Excellent performance for the separation of humic acid was obtained using the solution containing $0.5 \%(\mathrm{w} / \mathrm{v})$ HEC, $1.0 \%(\mathrm{w} / \mathrm{v})$ PEG 10000, and 0.1\% (w/v) PEG 8000000. Excellent reproducibility and durability were obtained even at slightly alkaline conditions at $\mathrm{pH}$ levels above 8 . The separation of $0.1-2 \mathrm{kbp}$ of DNA ladder by the proposed method showed was also achieved.
\end{abstract}

Keywords Capillary electrophoresis, dynamic coating, hydroxyethyl cellulose, polyethylene glycol, humic acid, DNA ladder

(Received August 5, 2013; Accepted September 5, 2013; Published November 10, 2013)

\section{Introduction}

Humic substances involve a variety of environmental issues and pose problems to the water supply industry. The substances require removal to minimize water color, and give rise to potentially mutagenic by-products as a result of chlorination. ${ }^{1}$ Their chemical alterations and interactions with coexisting materials are tightly involved with these issues. ${ }^{1-3}$ A great deal of research studies involving a varieties of analysis methods for humic substances, therefore, have been carried out. ${ }^{4-7}$

Separation techniques have been recognized as useful tools for the analysis and characterization of humic substances because they are a mixture of polymers with complicated compositions. There exists a large volume of work employing separation techniques to analyze and characterize them. ${ }^{8}$ Capillary electrophoresis $(\mathrm{CE})$ is one of the most promising techniques since it shows remarkable separation performance for the separation of natural polymers, such as proteins and nucleotides. ${ }^{9}$ In fact, studies on the helpfulness of $\mathrm{CE}$ for analyzing and characterizing humic substances have been published already. ${ }^{10,11}$ We previously reported a powerful CE separation technique for humic substances using a polyacrylamide-coated capillary and an electrophoretic buffer solution containing hydroxyethyl cellulose (HEC). ${ }^{12}$ In the method, electroosmotic flow (EOF) must be eliminated by immobilizing polyacrylamide on the capillary inner wall via silane coupling treatment followed by

$\dagger$ To whom correspondence should be addressed.

E-mail: toru@u-fukui.ac.jp in-capillary polymerization ${ }^{13}$ to avoid the delay of separation time since humic substances are polyanions with large electrophoretic mobility against the direction of EOF. Though the separation performance of the method is excellent, the procedure for preparing the polyacrylamide-coated capillary is troublesome. In addition, the capillary coating procedure is not always completed for all the capillaries prepared. Thus, a hassle-free and useful technique for coating the capillary inner wall is desired.

Along with the complicated procedure for preparation, attention must be paid to the cost, stability, and durability of the coated capillary. Another strategy for capillary coating to eliminate EOF other than "permanent" coating through covalent bonding is the dynamic coating method, ${ }^{14-16}$ and this method is quite promising from a practical point of view. The use of dynamic coating can significantly improve the routine performance of $\mathrm{CE}$ and the repeatability of migration time between capillaries. ${ }^{14,15}$ A quite simple dynamic coating method using a non-ionic polymer, such as polyethylene glycol (PEG) or HEC, has been established. ${ }^{17}$ The polymer was immobilized on the capillary inner wall via hydrogen bonding to the surface silanol groups by flushing the capillary with an acidic aqueous polymer solution after filling the capillary with $\mathrm{HCl}$ solution. While this procedure is quite simple, there is a problem that the coated capillary is only use in acidic and neutral conditions. It is recommended that the coated capillary for the analysis of humic substances in natural environmental water samples would be stable even at slightly basic conditions since the $\mathrm{pH}$ of natural water sometimes ranges over $8 .{ }^{18}$ Only the dynamically coated capillary prepared by the successive multiple ionic polymer 
layers technique ${ }^{19}$ is suitable for used in alkaline conditions, but the technique is not suitable for eliminating EOF. We present here a dynamic coating-based CE separation method for water soluble humic substances that improves operability, repeatability, and durability by employing an aqueous solution of mixed non-ionic polymers of different types and molecular weight as both the dynamic coating agent and the separation medium. The procedure for capillary treatment and CE analysis entails simply flushing the capillary with an acidic solution followed by filling the electrophoretic buffer solution containing polymers.

\section{Experimental}

\section{Reagents}

HEC was purchased from Wako Pure Chemical Industries Ltd. PEG 10000 and PEG 8000000 were both from Sigma-Aldrich Japan K.K. Commercially available humic acids (HA) were purchased from Sigma-Aldrich Japan and were hereafter referred to as (Aldrich HA). DNA ladder of $0.1-20 \mathrm{kbp}$ was purchased from Promega K.K. All other reagents used were of analytical grade.

\section{Procedure for CE separation}

CE separation was performed on an Agilent CE system with the constant voltage operation mode set at -15 or $-20 \mathrm{kV}$, using a GL Sciences (Tokyo, Japan) fused silica capillary (i.d., $50 \mu \mathrm{m}$, o.d., $330 \mu \mathrm{m})$. The total length $(L)$ and the effective length $(l)$ of the capillary used were 33.5 and $25 \mathrm{~cm}$, respectively. The capillary used was washed with aqueous solution of $0.1 \mathrm{M}$ $\mathrm{NaOH}$ followed by water. Then, the capillary was flushed by $1 \mathrm{M} \mathrm{HCl}$ aqueous solution followed by filling with an electrophoretic buffer solution containing $0.5 \%(w / v)$ HEC, $1.0 \%$ (w/v) PEG 10000, 0.1\% (w/v) PEG 8000000, and a pH buffer component. The procedures above were performed before every CE run. The polyacrylamide-coated capillaries were prepared in the same manner as described in an earlier paper. ${ }^{13}$ Sample solutions containing $0.5 \mathrm{mg} / \mathrm{mL}$ of humic substances were prepared with $10 \mathrm{mM}$ of tris(hydroxymethyl) aminomethane (Tris)/ $\mathrm{HCl}$ buffer ( $\mathrm{pH} \mathrm{8.0)}$. All the samples were filtrated by a Millipore (Bedford, MA) Millex syringe-driven filter unit (PTFE membrane, pore size $0.45 \mu \mathrm{m}$ ) before the $\mathrm{CE}$ run. A sample injection was performed electrokinetically at the anodic side of the capillary by applying a voltage $(-10 \mathrm{kV})$ for $10 \mathrm{~s}$. Direct photometric detection of $254 \mathrm{~nm}$ was performed at the anodic end of the capillary.

\section{Results and Discussion}

Dynamic coating CE with non-ionic polymers with hydrogen bonding ability

In the previous method, only HEC, which was used as the separation medium, was added to the electrophoretic buffer solution. ${ }^{12}$ On the other hand, it was found that the addition of PEG 10000 to the electrophoretic buffer solution slightly improved CE resolution. ${ }^{20}$ An electrophoretic buffer solution containing both HEC and PEG 10000 was, therefore, tested as the first candidate for the solution that served as both the separation medium and the dynamic coating agent in this study. Figure 1 shows the electropherograms of Aldrich HA using a polyacrylamide coating-fused silica capillary tube and the capillary prepared with dynamic coating procedure, both employing the same electrophoretic buffer solution containing HEC, PEG 10000, and $20 \mathrm{mM}$ phosphate buffer (pH 7.0). As

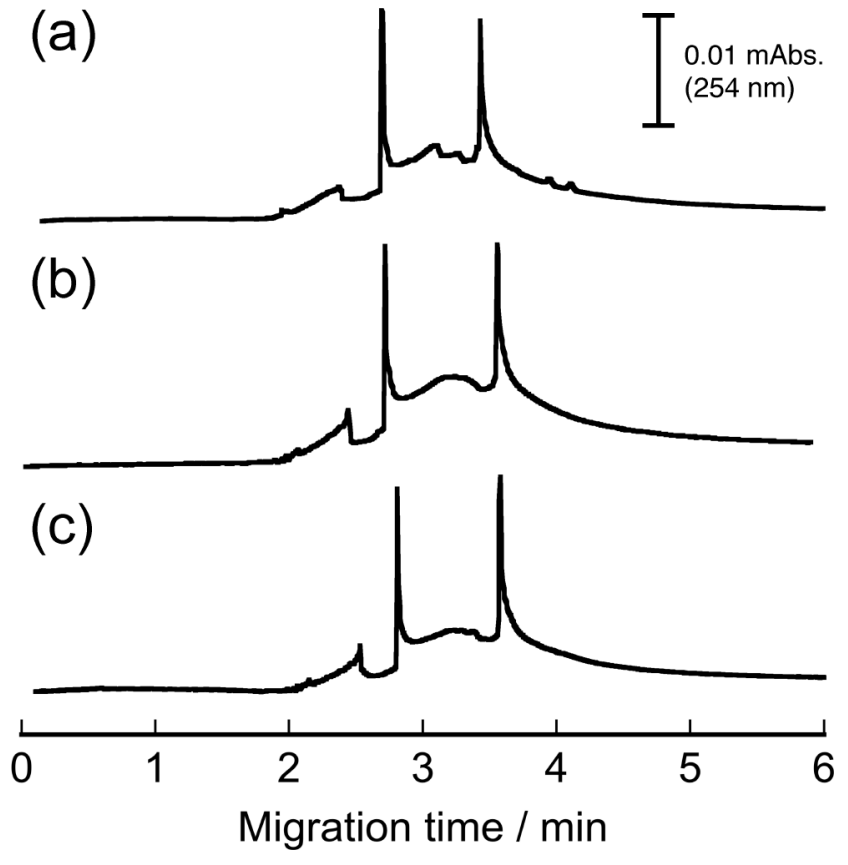

Fig. 1 Electropherograms of Aldrich HA. (a) Using polyacrylamide coating fused-silica capillary and an electrophoretic buffer solution (pH 7.0) containing $20 \mathrm{mM}$ phosphate, $0.5 \%$ HEC, and $1.0 \%$ PEG 10000; (b) using bare fused-silica capillary with dynamic coating procedure employing an electrophoretic buffer solution $(\mathrm{pH} 7.0)$ containing $20 \mathrm{mM}$ phosphate, $0.5 \%$ HEC, and 1.0\% PEG 10000; (c) using bare fused-silica capillary with dynamic coating procedure employing an electrophoretic buffer solution $(\mathrm{pH} 7.0)$ containing $20 \mathrm{mM}$ phosphate, $0.5 \%$ HEC, $1.0 \%$ PEG 10000, and 0.1\% PEG 8000000. Sample: $0.5 \mathrm{mg} / \mathrm{ml}$ Aldrich HA, $10 \mathrm{mM}$ Tris- $\mathrm{HCl}$ (pH 8.0). Capillary: $L=33.5 \mathrm{~cm}, l=25 \mathrm{~cm}$. Applying voltage: $-15 \mathrm{kV}$. Direct photometric detection of $254 \mathrm{~nm}$ was performed at anodic end of the capillary. The polyacrylamide-coated capillaries were prepared in the same manner as described in an earlier paper. ${ }^{13}$

seen in Figs. 1(a) and 1(b), exactly the same electropherograms were obtained by the previous method using a "permanent" polyacrylamide coating capillary (Fig. 1(a)) and the dynamic coating method (Fig. 1(b)). Though it was previously reported that the coated capillary with only HEC or PEG 8000000 via hydrogen bonding between polymers and protonated silanols on the surface of the capillary inner wall was stable only in weakly acidic conditions (below pH 6), ${ }^{17}$ the results of this work showed that the capillary coating was stable in neutral conditions, and EOF was completely eliminated at $\mathrm{pH} 7.0$ by employing the mixed polymer. The procedure for capillary coating in our previous method was complicated and took at least $3 \mathrm{~h}$. The procedure was drastically simplified in the proposed method as the coating is achieved just by flushing the electrophoretic buffer solution after washing with $\mathrm{HCl}$ solution for a few minutes.

For the analysis of humic substances in natural water samples, the capillary coating should be stable even at weakly alkaline conditions since the $\mathrm{pH}$ of natural water is in that range. In the CE separation of basic proteins using the neat electrophoretic buffer solution without polymers and the coated capillary with HEC and PEG by preliminary dynamic coating procedure, though the coated capillary of prepared with the solution containing only PEG 8000000 or PEG 10000 could be used only in lower $\mathrm{pH}$ conditions below $\mathrm{pH} 6$, that prepared with the solution containing both PEG 8000000 and PEG 10000 could 


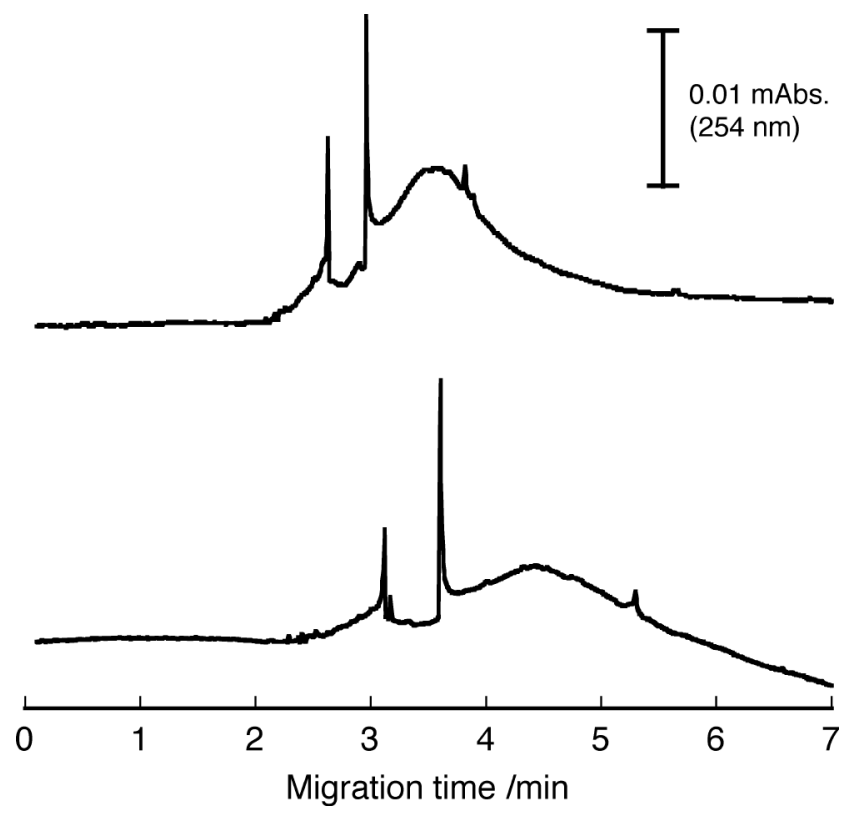

Fig. 2 Electropherograms of Aldrich HA by proposed method using an electrophoretic buffer solution of $\mathrm{pH} 8.0$ containing $20 \mathrm{mM}$ phosphate, $0.5 \%$ HEC, 1.0\% PEG 10000, and 0.1\% PEG 8000000 (a), and that without PEG 8000000 (b). Other conditions are the same as in Fig. 1(c).

be used in higher $\mathrm{pH}$ conditions around neutral. ${ }^{20}$ Therefore, the addition of PEG of large molecular weight was tested with the expectation that the capillary coating would become firm due to the tangle of polymer chains because it was found that the capillary coating with HEC and PEG 10000 was not stable in weakly alkaline conditions. The concentration of PEG 8000000 was determined by considering experimental operability since the solubility of PEG 8000000 in water is low and it takes a long time for it to completely dissolve in the solution. Figure 1(c) shows the electropherogram of HA using the electrophoretic buffer solution with the addition of $0.1 \%$ PEG 8000000. A comparison of the electropherogram in Fig. 1(c) with that of Fig. 1(b) shows that the addition of PEG 8000000 did not bring about a significant improvement in resolution, but an excellent electropherogram was obtained even in slight alkaline conditions. Figure 2 shows the electropherograms of Aldrich HA by the proposed method using the electrophoretic buffer solutions of $\mathrm{pH} 8.0$ with and without PEG 8000000 . Though the electropherogram patterns changed compared with those in Fig. 1 due to the conformational change of HA with the change in $\mathrm{pH}$, the general shape of both electropherograms in Fig. 2 is similar. A general increase of the migration time was observed in Fig. 2(b) as compared with Fig. 2(a). In this case, HA migrates from the negative end to positive end based on its own net electrophoretic mobility without receiving any resistance to opposite direction if the capillary inner wall is completely coated by polymers, as seen in Fig. 2(a). In the absence of PEG 8000000 (Fig. 2(b)), the increase of the migration time suggested that EOF arises with a breaking away of a part of the coating of the capillary inner wall with polymers because HA migrates to the positive end while encountering the resistance from the opposite direction due to EOF. The reason for the enhancement of the robustness of the capillary coating in slightly alkaline conditions with the addition of PEG 800000 would be that the entanglement of each polymer becomes
Table 1 Relative standard deviation (RSD) of the migration time for the first peak of the electropherogram of HA obtained by the proposed method at various $\mathrm{pH}$ levels.

\begin{tabular}{ll}
\hline $\mathrm{pH}$ & $\mathrm{RSD}, \%(n=5)$ \\
\hline 7.0 & $1.70\left(1.89^{\mathrm{a}}\right)$ \\
8.0 & $2.12\left(11.52^{\mathrm{a}}\right)$ \\
8.1 & 2.26 \\
8.2 & 1.99 \\
8.3 & 2.50 \\
8.4 & 4.41 \\
8.5 & 8.87 \\
\hline
\end{tabular}

a. proposed method without addition of PEG 8000000 .

firmer. Our previous method using a polyacrylamide coatedcapillary and the dynamic coating method without the addition of PEG 8000000 (Fig. 1(b)) cannot be used in conditions over pH 7 because EOF occurs slightly due to a partial breaking of the capillary coating. However, the dynamic coating method with the addition of PEG 8000000 can be used at $\mathrm{pH}$ 8.0. This composition of polymers, $0.5 \%(w / v)$ HEC, $1.0 \%$ (w/v) PEG 10000 , and $0.1 \%$ (w/v) PEG 8000000 , was used in all subsequent $\mathrm{CE}$ experiments.

\section{Stability of dynamically coated capillary}

CE experiments of 40 runs for the separation of Aldrich HA at pH 7.0 were performed. As seen in Fig. 1(c), in the typical electropherogram of Aldrich HA obtained by the proposed method, three sharp peaks were observed around 2.5, 2.8, and $3.7 \mathrm{~min}$, and a broad peak was seen around $3-3.5 \mathrm{~min}$. The migration time, $t_{\mathrm{m}}$, of the first peak of Aldrich HA (around $2.5 \mathrm{~min}$ ) with both the proposed method and our previous method was investigated. The value of $t_{\mathrm{m}}$ under the proposed method hardly changed through all $\mathrm{CE}$ runs. The relative standard deviation (RSD) of $t_{\mathrm{m}}$ through 40 runs was $1.95 \%$. In the case of using a polyacrylamide-coated capillary, the value of $t_{\mathrm{m}}$ clearly increased when the run number was over 15 . The increase in the value of $t_{\mathrm{m}}$ indicates the generation of EOF due to the avulsion of the polyacrylamide coating through repeated experiments. The proposed method showed significant improvement in routine $\mathrm{CE}$ experiments in turns of repeatability.

CE experiments for the separation of Aldrich HA by the proposed method at various $\mathrm{pH}$ levels were also performed. The RSD values of $t_{\mathrm{m}}$ for the first peak of Aldrich HA at $\mathrm{pH} 7.0-8.5$ were summarized in Table 1 . Adequately low RSD values of less than $5 \%$ were obtained under $\mathrm{pH} 8.4$, but RSD values were somewhat large at $\mathrm{pH} 8.5$. The proposed method can be applied to the analysis of humic substances in slightly alkaline conditions under $\mathrm{pH} \mathrm{8.4,} \mathrm{which} \mathrm{corresponds} \mathrm{to} \mathrm{the} \mathrm{pH}$ value of natural water samples, such as river water, and seawater. On the other hand, at $\mathrm{pH}$ 7.0, while an excellent RSD (1.89\%) was obtained without the addition of PEG 8000000 as was case with the addition of PEG 8000000, at $\mathrm{pH} 8.0$, RSD in the case without addition of PEG 8000000 deteriorated considerably to $11.52 \%$. The addition of PEG 8000000 to the electrophoretic buffer solution allowed for the excellent reproducibility of the $\mathrm{CE}$ experiments and the stability of the capillary coating at higher $\mathrm{pH}$ conditions.

\section{Separation of DNA ladder by the proposed method}

The separation of DNA ladder was also performed by the proposed method. Figure 3 shows the electropherogram of the DNA ladder of $0.1-20 \mathrm{kbp}$. Fourteen mutually separated peaks 


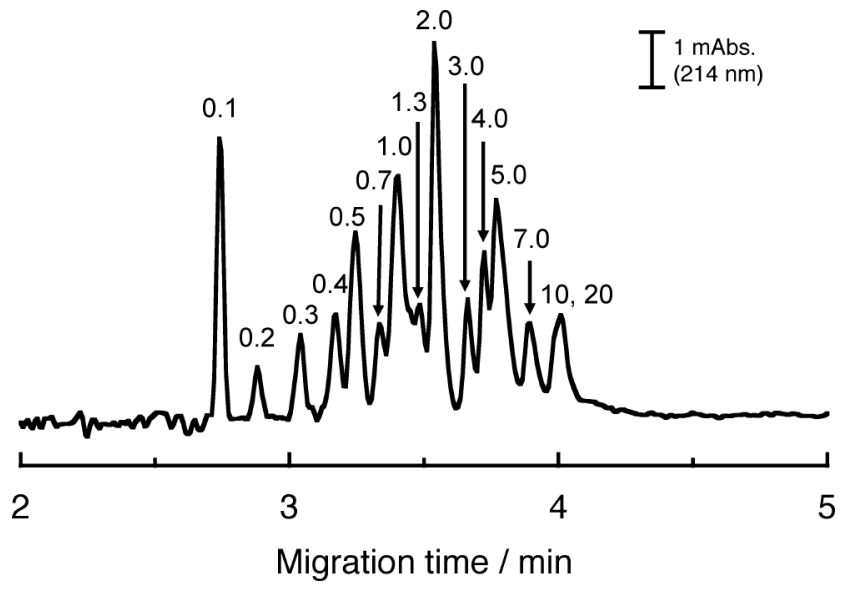

Fig. 3 Electropherogram of DNA ladder $(0.1-20 \mathrm{kbp})$ by proposed method. Direct photometric detection of $214 \mathrm{~nm}$ was performed at anodic end of the capillary. Other conditions are the same as in Fig. 1(c).

were observed, and 15 kinds of DNA with different chain length were separated mutually except 10 and $20 \mathrm{kbp}$. The results show that the proposed method would be applicable for the separation of mixtures of DNA with different chain length. The migration order of the proposed method corresponded to the order of the chain length of DNA, same as that of capillary gel electrophoresis based on the molecular sieving effect of the gel matrix filled into the capillary. ${ }^{20}$ It is, therefore, concluded that the separation of humic substances with the proposed method also would be essentially based on the molecular sieving effect of the mixed polymer matrix filled into the electrophoretic buffer solution.

\section{Conclusions}

A CE separation method for water-soluble humic substances with simple procedure and excellent separation performance was proposed. An aqueous solution of mixed non-ionic polymers with hydrogen bonding ability was used as both the dynamic coating agent for the fused-silica capillary inner wall and the molecular sieving medium for CE separation. The reproducibility of the proposed method is much higher than that of our previous method involving silane coupling treatment followed by polymerization reaction. High durability of the proposed method in slightly alkaline conditions above $\mathrm{pH} 8$ is noteworthy. Because the $\mathrm{pH}$ of natural waters sometimes ranges over 8 , the proposed method would be quite useful to characterize the state of humic substances in their usual $\mathrm{pH}$ condition in natural water samples. We previously found that the morphological change of humic substances due to the complexation with metal ion could be observed as the change of the electropherogram patterns. ${ }^{12}$ Of course, the proposed method would be applicable for the investigation of interactions of humic substances with coexisting materials including metal ions. The separation performance on the separation of $0.1-2 \mathrm{kbp}$ of DNA ladder by the proposed method showed that separation by the proposed method basically undergoes the molecular sieving effect of the mixed-polymer medium and that the proposed method was also applicable for the separation of analytes other than humic substances.

\section{Acknowledgements}

This work was supported in part by a research grant program of the Kurita Water and Environment Foundation (KWEF), Japan.

\section{References}

1. E. Tipping, "Cation Binding by Humic Substances", 2002, Cambridge University Press.

2. M. Schnitzer, Soil Sci., 1991, 151, 41.

3. N. Shinozuka, Seisan-kenkyu, 1993, 45, 486.

4. S. McDonald, A. G. Bishop, P. D. Prenzler, and K. Robards, Anal. Chim. Acta, 2004, 527, 105.

5. G. Abbt-Braun, U, Lankes, and F. H. Frimmel, Aquat. Sci., 2004, 66, 151.

6. M. Filella, Chem. Ecol., 2010, 26, 177.

7. L. A. Cardoza, A. K. Koir, W. H. Otto, C. J. Wurrey, and C. K. Larive, Prog. Nucl. Magn. Reson. Spectrosc., 2004, 45, 209.

8. P. Janos, J. Chromatogr., A, 2003, 983, 1.

9. S. R. Mikkelsen and E. Corton, "Bioanalytical Chemistry", 2004, Chap. 12, John Wiley \& Sons, Ltd.

10. P. Schmitt-Kopplin and J. Junkers, in "Environmental Colloids and Particles: Behavior, Separation and Characterization", ed. K. J. Wilkinson and J. R. Lead, 2007, John Wiley \& Sons, Ltd., 277.

11. P. Schmitt-Kopplin and J. Junkers, J. Chromatogr., A, 2003 , 998, 1.

12. T. Takahashi, J. Kawana, and H. Hoshino, Anal. Bioanal. Chem., 2009, 393, 761.

13. C. Sumita, Y. Baba, K. Hide, N. Ishimaru, K. Samata, A Tanaka, and M. Tsuhako, J. Chromatogr., A, 1994, 661, 263.

14. K. D. Altria, LC-GC Europe, 2012, 25, 201.

15. K. D. Altria, J. Pharm. Biomed. Anal., 2003, 31, 447.

16. J. Znaleziona, J. Petr, R. Knob, V. Maier, and D. J. Svevcik, Chromatographia, 2008, 67, S5.

17. N. Iki and E. S. Yeung, J. Chromatogr., A, 1996, 731, 273.

18. R. N. Reeve, "Introduction to Environmental Studies", 2002, Chap. 3, John Wiley \& Sons, Ltd.

19. H. Katayama, Y. Ishihama, and N. Asakawa, Anal. Chem., 1998, 70, 2254.

20. J. Kawana, Master Thesis, Tohoku University, 2009. 